Archives

18-19 | 1997

Inventaire des archives de Robert Mandrou

\title{
République des lettres, intellectuels laïcs et mécénat princier
}

\section{Roger Chartier}

\section{OpenEdition}

Journals

Édition électronique

URL : http://journals.openedition.org/ccrh/2570

DOI : $10.4000 /$ ccrh. 2570

ISSN : $1760-7906$

Éditeur

Centre de recherches historiques - EHESS

Édition imprimée

Date de publication : 10 octobre 1997

ISSN : 0990-9141

Référence électronique

Roger Chartier, « République des lettres, intellectuels laïcs et mécénat princier », Les Cahiers du Centre de Recherches Historiques [En ligne], 18-19 | 1997, mis en ligne le 20 février 2009, consulté le 19 avril 2019. URL : http://journals.openedition.org/ccrh/2570; DOI : 10.4000/ccrh.2570

Ce document a été généré automatiquement le 19 avril 2019

Article L.111-1 du Code de la propriété intellectuelle. 


\title{
République des lettres, intellectuels laïcs et mécénat princier
}

\author{
Roger Chartier
}

1 Robert Mandrou a inventé plusieurs des grands sujets qui ont mobilisé le travail des historiens depuis trente ans. Aujourd'hui encore, tous les travaux menés sur la sorcellerie ou la culture populaire ne peuvent que partir, même si c'est pour s'en séparer, des constats et des hypothèses proposés par Magistrats et sorciers ou De la culture populaire. Mais, à côté de ces œuvres pionnières et, pour cela même, risquées, il en a publié d'autres, plus nombreuses au demeurant, dont la facture classique ne doit pas faire sous-estimer l'originalité.

2 Tout au long de son parcours d'historien, Robert Mandrou a, en effet, rédigé des synthèses de grande ambition, destinées aux étudiants et à ses collègues professeurs, mais aussi à un public plus large de lecteurs d'histoire. Cinq livres jalonnent cette trajectoire : en 1958, chez Armand Colin, l'Histoire de la civilisation française, rédigée en collaboration avec Georges Duby; en 1961, dans la collection «L'Évolution de l'humanité » d'Albin Michel, l'Introduction à la France moderne, essai de psychologie historique (qui sera rééditée à l'automne de cette année dans la «Bibliothèque de l'Évolution de l'humanité » avec une préface de Jean Lecuir et Philippe Joutard qui situera l'ouvrage dans le contexte difficile de sa publication); en 1967, le volume 33 de la collection " Nouvelle Clio » La France aux $\mathrm{XVII}^{\mathrm{e}}$ et XVIII ${ }^{\mathrm{e}}$ siècles; et, en 1973, Louis XIV en son temps, dans la vénérable collection des PUF «Peuples et civilisations» fondée par Halphen et Sagnac, et Des humanistes aux hommes de science (XVI et XVII siècles), tome troisième d'une Histoire de la pensée européenne publiée en édition de poche par Le Seuil.

3 Le sort de telles synthèses n'est pas sans injustice. Leur statut de références obligées, souvent citées dans les bibliographies, a parfois effacé la nouveauté qui était la leur au moment de leur publication. Les relire aujourd'hui est mesurer, à la fois, les déplacements du travail des historiens en ces trente dernières années et l'acuité avec laquelle Robert Mandrou désignait et dessinait les directions de recherche aptes à renouveler ce qu'il nommait, avec des termes qui pour lui étaient presque équivalents, "histoire des mentalités », « histoire des psychologies collectives » ou « histoire culturelle ». Contre les 
présupposés anachroniques d'une " caractérologie moralisante et éclectique » dotant les hommes et les femmes du passé des sentiments, des intentions et des émotions de ceux d'aujourd'hui, contre les interprétations anhistoriques des idées et des œuvres, Robert Mandrou plaidait pour l'historicité, donc la discontinuité des horizons mentaux et des visions du monde. Fidèle aux leçons de Lucien Febvre, il assignait à l'histoire culturelle la tâche de reconstituer les pensées, les croyances et les rêves propres à chaque groupe social (noblesse de robe, bourgeoisie marchande, milieux populaires), à chaque milieu professionnel (officiers, diplomates, hommes de loi, artisans, artistes, etc.) ou à chaque communauté intellectuelle (par exemple les humanistes, les libertins érudits, les savants, etc.).

\section{République des lettres et révolution scientifique}

Dans La France aux XVII et XVIII ${ }^{\mathrm{e}}$ siècles, parue il y a trente ans, Robert Mandrou marquait les renouvellements majeurs qui avaient, selon lui, transformé la connaissance de l'«Ancien Régime socioculturel ». Il les rangeait sous trois catégories : les enquêtes de la sociologie religieuse rétrospective, l'étude des différents milieux socioculturels, l'exploration thématique et sérielle de la production imprimée. Les monographies inspirées par l'œuvre de Gabriel Le Bras, à commencer par celle de Louis Perouas sur le diocèse de La Rochelle, le livre de René Pintard sur les libertins érudits, les thèses d'histoire littéraire sur les idées de bonheur (celle Robert Mauzi) et de nature (celles de Jean Ehrard et de Jacques Roger), ou encore l'enquête Livre et société dans la France du XVIII ${ }^{e}$ siècle, donnaient support à ce diagnostic. Les analyses qu'il fondait ont conservé toute leur pertinence-sauf, sans doute, celle qui caractérise trop uniment comme «bourgeoises » les pensées et les sociabilités nouvelles des Lumières et celle qui identifie trop hâtivement la culture et la sensibilité populaires à partir des textes qui composent le répertoire de la littérature de colportage.

5 Tourné vers ce qui restait à faire dans le domaine de l'histoire culturelle, Mandrou privilégiait cinq thèmes de recherche: le respect - ou le refus - des valeurs sociales dominantes, les pratiques et les sentiments du vécu chrétien, les modèles éducatifs et les méthodes d'enseignement, les supports et motifs de l'information, les publics artistiques (de la peinture, du théâtre, de l'opéra). Très largement, le travail des historiens a ratifié ses choix et pour chacun des thèmes qu'il indiquait les travaux se sont multipliés, répondant au programme tracé à partir de la lecture d'ouvrages pionniers (ceux de Foucault et d'Ariès), de l'avancement des enquêtes collectives dirigées par Alphonse Dupront ou des premiers travaux exploitant les archives judiciaires.

6 En un temps où commençait l'engouement pour l'histoire sérielle de la culture, Robert Mandrou en marquait, comme préventivement, l'apport et les limites. Soit l'exemple de la circulation de l'imprimé. Il soulignait l'importance des études attachées «surtout à la production, aux tirages et rééditions, quand les documents en fournissent les données, et à la ventilation des genres ", mais, dans le même temps, il en suggérait le possible et nécessaire dépassement : "Mémoires, livres de raison, critiques viennent au secours du chercheur qui veut se représenter celui qui lit, voire pourquoi il lit - et non seulement celui qui écrit et se fait imprimer. » Était ainsi indiqué en filigrane le déplacement qui, plus tard, a conduit de l'histoire de la production et de la circulation du livre à celle des pratiques de lecture. 
7 «Assemblées, correspondances et voyages constituent les trois supports de l'information savante » : cette formule, extraite du livre Des humanistes aux hommes de science, illustre le souci constant chez Mandrou de rapporter le travail de la pensée à ses conditions de possibilité. Relu à la lumière des travaux les plus récents, l'ouvrage, publié en 1973, frappe par l'accent (alors très neuf) mis sur les pratiques sociales et intellectuelles constitutives de la république des lettres en ses diverses modalités. Les sodalitates literarum du temps de l'humanisme, les compagnies érudites du premier xvII siècle, les académies londonienne et parisienne fondées dans la décennie 1660 sont trois figures majeures - et différentes en leur assise et leur fonctionnement - des solidarités qui autorisent l'émancipation des "nouveaux intellectuels" vis-à-vis des institutions religieuses et universitaires traditionnelles. Anticipant sur les caractérisations les plus récentes de la république des savants, Robert Mandrou soulignait avec force le rôle joué par une éthique de l'entraide, de l'hospitalité et de la réciprocité dans la constitution d'un monde intellectuel engagé dans un processus d'autonomisation. Les échanges multiples (de lettres, de livres, de manuscrits, prêtés ou copiés, d'informations) permis par les correspondances, les voyages et les visites incarnent, dans les pratiques ordinaires, l'idéal d'un univers soustrait aux contraintes et aux dominations exercées par les autorités anciennes.

Robert Mandrou marquait également les limites durables de cette indépendance difficilement affirmée : «Ce qui manque le plus encore à la fin du XVII e siècle, c'est l'appui d'un public, qui assurerait économiquement la place de ces nouveaux clercs dans la société. » De là, les choix obligés qui s'imposent à eux : soit entrer dans des emplois leur permettant de subsister - ainsi au Xvi ${ }^{\mathrm{e}}$ siècle ceux de correcteur ou de pédagogue, et au XVII ${ }^{e}$ siècle ceux proposés par le service du prince ou de l'administration de l'État -, ou bien prendre place dans la clientèle d'un roi ou d'un grand et vivre des gratifications du mécénat. Le prix à payer, dans ce cas, n'est pas mince, impliquant le recours aux formes multiples de l'écriture de célébration, de la dédicace à l'éloge.

La conclusion du livre, voué à repérer les premières figures de l'intellectuel «moderne », insiste sur la précarité et l'incertitude du statut qui est le sien entre $\mathrm{XVI}^{\mathrm{e}}$ et $\mathrm{XVII}^{\mathrm{e}}$ siècle : "Les transformations du monde pendant ces deux cents ans ont consacré l'éclatement du cadre ecclésial, mais n'ont pu, d'elles-mêmes, susciter un nouveau cadre aussi bien délimité que l'ancien.» De là, l'ambiguïté de la position des premiers «intellectuels laïcs ", libérés des nécessaires disciplines de l'Église, mais soumis aux contraintes du mécénat et de la protection princière. Une telle analyse, qui porte la construction même du livre, permet de penser comment la dépendance acceptée à l'égard du roi, de la cour ou de l'État est la condition même pour que puisse prendre réalité (au moins partiellement) l'émancipation par rapport à l'Université (dans le cas des hommes de science) ou par rapport aux communautés de métier (dans le cas des artistes). Il est frappant de constater que les recherches les plus récentes sur les lieux et les conditions mêmes de la pratique scientifique (avec Galilée), littéraire (avec Shakespeare, Lope de Vega ou Corneille) ou picturale (avec les Carrache ou Rubens) se sont attachées, avant tout, à comprendre les effets de ce jeu complexe qui inscrit dans les obligations réciproques du patronage, les libertés conquises aux dépens des autorités et des corps anciens.

Robert Mandrou avait discerné avec lucidité les effets intellectuels d'une telle ambiguïté. Certes, il affirmait en conclusion de son livre : «Sans nul doute, les affrontements qui ont rythmé le devenir de la pensée européenne pendant ces deux siècles mettent bien en 
présence des camps antagonistes, les défenseurs vigilants d'orthodoxies contestées et les cohortes des novateurs » - et il ne fait pas de doute que ses sympathies allaient vers tous ceux qui avaient mis à mal, parfois au prix de leur vie, les pensées et les croyances héritées. Mais il ajoutait aussitôt que la ligne de partage entre les gardiens de la tradition et les esprits critiques n'était pas aussi simplement dessinée qu'on l'avait longtemps cru. Elle traversait chaque institution - à commencer par l'Église catholique -, chaque milieu, chaque individu. D'où le diagnostic final : « La polarisation tentante qui situerait tous les penseurs du temps sur l'un ou l'autre axe, la défense de l'ordre établi, suivant un principe de régulation sociale nécessaire d'un côté, l'attaque de cet ordre et la définition d'un autre ordre à venir, à créer de l'autre côté, n'est donc pas tout à fait satisfaisante. »

11 C'est à partir de cette insatisfaction devant des oppositions trop brutales que Robert Mandrou a construit la description, parfois rapide mais toujours subtile et informée, des courants dominants ou marginaux de la vie intellectuelle européenne entre 1480 et 1700 . Plusieurs traits seraient aujourd'hui accusés différemment. Retenons-en trois, non pas comme des critiques d'un livre qui ne pouvait qu'utiliser les travaux alors disponibles, mais comme exemples des déplacements du savoir historique. Et tout d'abord les pages consacrées à "l'apparition du livre" identifiée à l'invention et à la diffusion de l'imprimerie, qui assure « le passage de la parole et de l'image gravée ou peinte à l'écrit imprimé, reproduit par centaines d'exemplaires mettant à la disposition d'un public large un texte ne varietur, nettoyé des fautes de lassitude commises par les copistes, et conservés pendant des années ». Réécrite aujourd'hui, une telle description, qui reprend les thèmes et jusqu'au titre (malheureux) du livre de Febvre et Martin publié en 1958, insisterait bien plus sur les limites de la « révolution de l'imprimé ». D'une part, le livre n'apparaît pas avec Gutenberg et sa structure fondamentale, celle du codex, inventé aux premiers siècles de l'ère chrétienne, demeure la même après comme avant l'invention de l'imprimerie. D'autre part, loin d'assurer la parfaite stabilité du texte, le processus même de production et de fabrication du livre imprimé multiplie les variantes, non seulement entre les éditions d'une même œuvre en un temps où les tirages demeurent limités, mais aussi, du fait des corrections en cours de tirage, entre les exemplaires d'une même édition. Enfin, le recours à la publication manuscrite reste essentiel entre $\mathrm{XV}^{\mathrm{e}}$ et $\mathrm{XVIII}^{\mathrm{e}}$ siècle. C'est elle qui fonde les pratiques de la république des lettres, dont les membres échangent lettres, mémoires et copies manuscrits, s'efforçant ainsi d'éviter la corruption des textes qu'introduisent les erreurs de compositeurs maladroits, la cupidité des libraires-éditeurs ou les interprétations incontrôlées de lecteurs ignorants. Loin de porter l'éthique propre à la république des lettres, l'imprimerie en est, au contraire, l'adversaire redouté.

Dans les pages qu'il consacre aux démarches intellectuelles des érudits et savants du premier $\mathrm{xvII}^{\mathrm{e}}$ siècle, Robert Mandrou porte l'accent sur «la lenteur démonstrative par accumulation de références »: "Chacun décrit, rappelle et cite des sources anciennes bien connues; chacun expérimente ou observe, avec finesse souvent ; parfois avec autant de crédulité qu'il en est refusé deux pages auparavant. Mais autant de citations, de constats ne s'additionnent pas; parfois même se contredisent, sous la même plume, à quelques années d'intervalle. » Le jugement est tout à fait perspicace. Il serait maintenant formulé d'une autre manière, considérant que ces manières d'écrire et de raisonner définissent une technique intellectuelle spécifique, celle des loci communes. Plusieurs travaux récemment publiés ont montré la cohérence et la prégnance de rapport particulier à la culture écrite qui nourrit la nécessaire copia des textes rédigés ou 
prononcés à partir des citations, des exemples et des modèles que l'auteur extrait des cahiers et des livres de «lieux communs » où se trouvent redistribués thématiquement, par topiques et rubriques, des fragments des ouvrages consultés et compilés. Il y a là le fondement d'une manière de lire, de dire et d'écrire qui met l'accumulation de références et de citations au service tant de l'amplification rhétorique que de l'argumentation démonstrative.

De façon parallèle, les différences notées par Robert Mandrou entre le modèle logicomathématique de la connaissance (tel que le porte le cartésianisme) et le primat donné à l'expérience et aux applications pratiques (tel qu'on le rencontre à la Royal Society) pourraient, à la lumière des travaux récents en histoire des sciences, être formulées de manière plus fortement contrastée comme deux définitions de la "révolution scientifique ». La première, galiléo-cartésienne, identifie le nouveau savoir au déchiffrement de la nature écrite en langage géométrique. La seconde, dont l'épicentre se situe en Angleterre, appuie sa description du monde sur la philosophie naturelle expérimentale. Plus peut-être que ne le pensait Mandrou, qui réunit les deux démarches dans un même "esprit scientifique", chaque conception implique un modèle épistémologique, des pratiques savantes et des institutions de savoir très différents.

Ces quelques remarques ont pour but de montrer qu'un livre est toujours inscrit en son temps. Robert Mandrou a pressenti avec un discernement rare quelques-unes des révisions historiographiques les plus importantes de ces trente dernières années. Faute de travaux pouvant appuyer alors ses intuitions, les formulations qui sont les siennes peuvent apparaitre comme inachevées ou trop prudentes. Elles n'en indiquent pas moins des thèmes et des directions de recherche qui ont, après lui, largement renouvelé l'histoire culturelle.

\section{Mécénat princier et liberté savante}

Ce sont ces mêmes thèmes et ces mêmes orientations qui font l'originalité des développements consacrés aux formes de la vie intellectuelle et aux pratiques culturelles dans Louis XIV en son temps. Dans chacune des deux grandes parties qui composent le livre et que sépare la césure de 1685 , le sixième chapitre leur est consacré. Cette symétrie n'est pas aléatoire puisqu'elle oppose terme à terme, comme dans un diptyque, « la direction des lettres et des arts ", qui contraint, par la censure, la surveillance et le mécénat, la production intellectuelle et artistique dans la France des années 1661-1685, et «la vie culturelle en Angleterre à la fin du XvII ${ }^{\mathrm{e}}$ siècle » que caractérise le choix des libertés et des sciences.

Mandrou insiste, du côté français, sur l'importance de la commande royale et des représentations à la cour, anticipant ainsi sur les réévaluations qui ont replacé dans leur cadre premier des œuvres trop hâtivement considérées comme adressées au public citadin. Il en va ainsi des pièces de Molière, dont Mandrou rappelle qu'à partir de 1663 elles ont été offertes "d'abord aux applaudissements des courtisans » avant d'être reprises sur la scène du théâtre du Palais-Royal. Désirant prendre mesure des traductions financières du mécénat louis-quatorzien, il utilise les listes des pensions et gratifications accordées aux gens de lettres telles qu'elles figurent dans les Comptes des bâtiments $d u$ roi (et telles qu'elles ont été publiées par Pierre Clément en 1868 puis par Jules Guiffrey en 1881). 
17 Les conclusions sont nettes - et n'ont sans doute pas été suffisamment exploitées et prolongées. Elles tiennent en quatre constats essentiels :

1. La munificence royale décroît fortement à partir de 1674 du fait des dépensées liées à la guerre de Hollande: alors qu'entre 1664 et 1673 la moyenne des sommes distribuées s'élève à 95000 livres, elle tombe 57000 livres dans les dix années suivantes et plus aucune gratification n'est adressée à l'étranger ;

2. La part des pensions et gratifications ne représente qu'un pourcentage infime du budget de la monarchie (de $0,1 \%$ à $0,05 \%$ ) mais il est vrai qu'elle ne peut être tenue pour la totalité des paiements faits en faveur des écrivains, des artistes et des savants;

3. Le nombre des bénéficiaires est toujours demeuré restreint et a été réduit après 1674 (une cinquantaine plus une douzaine d'étrangers avant cette date, entre trente et quarante après), ce qui fait que la moyenne des gratifications reste stable durant toute la période (autour de 1600 livres entre 1667 et 1676) et que, pour ceux qui se retrouvent sur les listes d'année en année, « la gratification royale est devenue une sorte de rente, payée à peu près régulièrement, et assez confortable ";

4. La protection royale concerne majoritairement des hommes de lettres (historiens, prédicateurs, théologiens, dramaturges, poètes), même si la présence des savants se fait plus forte aux débuts de la décennie 1670 avant de retomber ensuite. Les données ainsi rassemblées invitent à reconsidérer de manière neuve la question classique de l'enrôlement des écrivains et des savants au service de la gloire du roi. Elle conduit à repérer dans les œuvres elles-mêmes les effets des contraintes qui ont, tout ensemble, borné et demandé leur production. Dans ces pages de son Louis XIV, Mandrou a pressenti avec intelligence les nouvelles voies que devait suivre une histoire des genres et des textes soucieuse de ne pas séparer la compréhension de leurs significations et l'analyse de leur esthétique de l'étude des exigences et des urgences qui les gouvernent.

$\mathrm{Au}$ contrôle exercé par le monarque français sur la vie littéraire et scientifique, Mandrou oppose la liberté anglaise. Le contraste ne doit pas être forcé dans la mesure où la direction des lettres et des arts n'a jamais complètement étouffé, et moins encore dans les vingt-cinq dernières années du règne, le mécénat des particuliers, les formes privées du débat intellectuel ou la sociabilité amicale et choisie des salons parisiens. Mais ce qui reste comme une différence essentielle est l'autonomie accordée en Angleterre à l'institution académique elle-même, en l'occurrence la Royal Society. "Aucune autre assemblée scientifique n'a cumulé à l'époque autant de privilèges que la Société Royale : liberté de recrutement, engouement constant, aisance matérielle, liberté dans le choix des travaux comme dans l'établissement des relations avec l'étranger. »

Cette " liberté ", permise à la Royal Society mais non pas aux académies royales françaises, était pour Robert Mandrou la condition même du travail intellectuel - au $x^{e}$ siècle comme au XVII ${ }^{e}$. Celui-ci suppose l'échange, la collaboration, l'entraide, noués par-delà les frontières et en dépit, ou plutôt à cause des différences. Dans les sociétés d'Ancien Régime, la correspondance a été durablement l'instrument privilégié de la réciprocité entre les lettrés. Ce n'est donc pas par hasard que, dans deux des ouvrages que nous avons commentés ici, trois cartes donnent à voir l'espace européen ouvert et tolérant, ignorant les divisions entre les religions et les conflits entre les États, qui a été celui de trois grands épistoliers de l'Âge moderne : Erasme, Peiresc, Leibniz. Chacun à sa façon, chacun en son temps, illustre magnifiquement les valeurs propres de la république des lettres - cette république dont Robert Mandrou se pensait et voulait citoyen. 